\title{
ILCEA
}

Revue de l'Institut des langues et cultures

d'Europe, Amérique, Afrique, Asie et Australie

44 | 2021

Peinture, identité nationale et style international en

Europe autour de 1900

\section{L'Exposition universelle de 1900 et les apories d'une « école italienne »}

1900 World's Fair and the Italian School's Inconsistency

L'Esposizione Universale del 1900 e le aporie della «scuola italiana»

Marion Lagrange

\section{OpenEdition}

Journals

Édition électronique

URL : https://journals.openedition.org/ilcea/12518

DOI : 10.4000/ilcea.12518

ISSN : 2101-0609

Éditeur

UGA Éditions/Université Grenoble Alpes

Édition imprimée

ISBN : 978-2-37747-324-3

ISSN : $1639-6073$

\section{Référence électronique}

Marion Lagrange, «L'Exposition universelle de 1900 et les apories d'une « école italienne » », ILCEA [En ligne], 44 | 2021, mis en ligne le 02 novembre 2021, consulté le 03 décembre 2021. URL : http:// journals.openedition.org/ilcea/12518; DOI : https://doi.org/10.4000/ilcea.12518

Ce document a été généré automatiquement le 3 décembre 2021

(C) ILCEA 


\title{
L'Exposition universelle de 1900 et les apories d'une « école italienne »
}

\author{
1900 World's Fair and the Italian School's Inconsistency \\ L'Esposizione Universale del 1900 e le aporie della «scuola italiana»
}

\author{
Marion Lagrange
}

1 En 1886, La Nouvelle Revue, dirigée par la progressiste Juliette Adam, publia un long et inattendu état des lieux de la peinture italienne sous la plume d'une certaine Lotty Milliot. Le "progrès» observé notamment lors de la dernière exposition nationale des beaux-arts organisée à Turin, l'amenait à s'intéresser aux mérites de plusieurs artistes sans qu'ils puissent, selon elle, prétendre à former une "école». Lucide, l'auteure mettait néanmoins en garde le lecteur contre toute forme de nostalgie : «Mais, entendons-nous. Il ne s'agit plus des anciennes écoles. [...] Elles ont disparu ces écoles romaines et florentines, vénitiennes et bolonaises, créations des Véronèse, des Carrache, des Michel-Ange, des Raphaël [...]. Le monde marche, il va de l'avant, et, sans renier le passé, il doit faire du nouveau » (Milliot, 1886). Une dizaine d'années après la publication par Charles Blanc de "son " histoire ${ }^{1}$ - où il n'était encore question que des écoles vénitienne, bolonaise, florentine, romaine et ombrienne (et de leurs maitres) - une rupture semblait s'opérer dans le continuum du récit historique construit sur le polycentrisme de l'art italien (Castelnuovo \& Ginzburg, 1981 : 52). L'idée d'une école italienne en concordance avec les aspirations unitaires et réformatrices du Risorgimento, tendait à s'imposer.

2 Cette approche, issue d'une conception traditionnelle de la géographie artistique (Gamboni, 1987 ; Dacosta Kaufmann, 2004), encourageait à définir des unités artistiques homogènes en fonction d'un territoire. Depuis 1855, la notion d'école (Peltre \& Lorentz, 2007) avait pris une acuité particulière sous la plume des critiques d'art dans le cadre international et identitaire des accrochages, sous «pavillon national », des Expositions universelles. Huit ans après l'avènement, en 1870, du nouvel État-nation, on encourageait les développements de l'école italienne. Le terme peinait pourtant encore à s'imposer en 1889, si ce n'est pour en déplorer la faiblesse. Paradoxalement, en 1900, alors que le «palais vénitien » se dressait aux abords de la Seine, le comité italien de 
l'Exposition y présentait un panorama de l'art ancré dans la territorialité et structuré autour de centres artistiques spécifiques (Picone Petrusa, 2015). La notion d'école italienne se trouvait alors moins investie que celle des écoles régionales dont la modernité s'apparentait à une affirmation des spécificités identitaires, révélant a priori en creux l'effacement de la figure du maître. Ce sujet, replacé dans un cadre historiographique plus large, mérite toute notre attention. Les problématiques liées à la réception de l'œuvre, à la construction des discours et aux transferts culturels, en œuvre dans le cadre des expositions internationales, ont fait l'objet de maintes recherches (Forster-Hahn, 2003 ; Ruedin, 2010). Parallèlement, les liens de l'idéologie nationale avec l'histoire de l'art ou la critique ont été particulièrement examinés (Roland \& Schmitz, 2004; Hargrove \& McWilliam, 2005 ; Laugée \& Rabiller, 2017). En Italie, la question identitaire a été étudiée tant par le biais des expositions nationales et internationales que par celui des débats artistiques portant sur l'identité dans l'espace de la péninsule (Lamberti, 1982 ; Poppi, 1988 ; Picone Petrusa, Pessolano \& Bianco, 1988; Maggio Serra, 1991 [1990]; Ceschin, 2001; Lamberti, 2005 ; Della Coletta, 2006). Exception faite de la sculpture (Pingeot, 2000), l'art italien au sein des expositions internationales à l'étranger demeure encore peu exploré ${ }^{2}$.

4 Si l'on considère l'Exposition universelle de 1900 comme un laboratoire des problématiques identitaires de la peinture italienne, celle-ci apparait comme un terrain d'étude privilégié de la dialectique active entre nation et région, la notion d'école en constituant le fil conducteur. Dans cette perspective, la vision stéréotypée et les préjugés nationaux diffusés dans la presse française nous intéresserons moins que la manière dont les permanences historiographiques influencent la conception d'un art italien replacé dans un récit européen et moderniste.

\section{Parisianisme versus « italianité »}

5 L'Exposition du Siècle marqua indéniablement un jalon stratégique où les artistes aspiraient encore à une reconnaissance parisienne, sésame pour une carrière artistique nationale ou internationale alors que l'Esposizione Internazionale d'Arte de Venise ne donnait pas encore satisfaction (Lamberti, 1982 : 111-116 ; Bruno, 2010 : 145-182). La section italienne se composait de cent vingt-et-un exposants, soixante-quatorze peintres et dessinateurs et quarante-sept sculpteurs, pour un total de deux cent seize œuvres. L'ensemble était exposé dans le Grand Palais, nouvel édifice réservé à la présentation des beaux-arts (fig. 1). 
Fig. 1. - Disposition intérieure du Grand Palais des beaux-arts, reproduit dans Quantin, $1900: 22$.

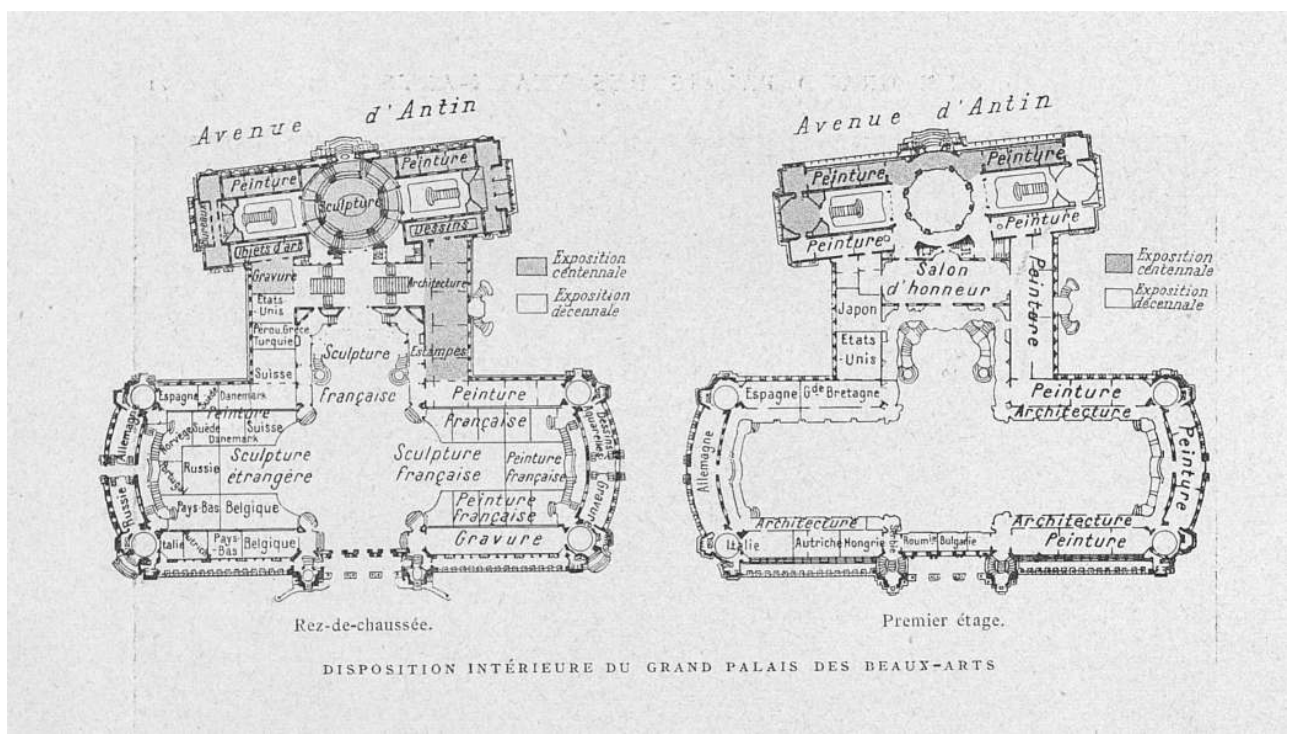

(c) Source gallica.bnf.fr /BnF. elles sont significatives des tensions alors existantes entre les «Italiens d'Italie » et les «Italiens de Paris».

7 Afin de mieux saisir les enjeux de cette dichotomie, il n'est pas inutile de revenir sur l'organisation de la section artistique italienne de l'Exposition universelle de 1889. Celle-ci avait été la vitrine d'un art national produit à l'intérieur et à l'extérieur de l'Italie. Pour différents motifs ${ }^{3}$, cette manifestation avait été le fruit d'une cotutelle entre un comité national et un comité «italo-parisien». Une association d'artistes installés à Paris s'était formé, d'abord sous la houlette du peintre orientaliste Alberto Pasini (1826-1899) [Anonyme, 1888], grand prix de l'Exposition universelle de 1878, puis de Giovanni Boldini (1842-1931) [Anonyme, 1889], et ce afin d'assurer leur place dans les manifestations internationales. Pour la première fois, un peintre ayant fait carrière à Paris occupait cette fonction. Boldini succédait notamment à Eleuterio Pagliano (1826-1903) [J. M., 1878] pour 1878 et Domenico Morelli (1826-1901) pour 1867 (Anonyme, $1867: 10$ ). Le gouvernement italien, reprenant la main sur l'organisation de l'Exposition universelle de 1900, mit fin à cette situation qui avait pu se justifier en 1889. Boldini fut néanmoins désigné comme membre du jury d'admission de la section internationale de la classe des beaux-arts (Anonyme, 1900a).

8 Cette relative mise à l'écart des «Italiens de Paris » du comité d'organisation ne les empêcha pas d'être particulièrement remarqués lors des festivités de l'inauguration (Brémond, 1900) :

Hier, à cinq heures et demie, on a inauguré ce merveilleux palais [...]. La fête était présidée par M. Salandra, ministre du commerce d'Italie, qui était venu à Paris spécialement pour cette inauguration. Le ministre était accompagné par M.le comte Tornielli, ambassadeur d'Italie [...]. Parmi les personnes qui l'entouraient nous avons reconnu: [...] le peintre Boldini, M. Camondo, Mlle Juana Romani, le sculpteur Gallori, le peintre To[f]ano, l'auteur du tableau si connu : «Enfin seuls!» [...]. célébrités de la scène parisienne, régulièrement présents aux Salons ${ }^{4}$. Seule femme avec 
la sculptrice naturalisée Italienne Marcelle Lancellot-Croce (1863-1946), Juana Romani (1867-1923), pouvait se prévaloir d'exposer deux tableaux, Primavera et Salomé (fig. 2), déjà acquis par l'État lors du Salon des artistes français, respectivement en 1895 et 1898.

Fig. 2. - D'après Juana Romani, Salomé, 1898, carte postale du musée du Luxembourg ( $\left.{ }^{\circ} 149\right)$, $6 \times 9 \mathrm{~cm}$, coll. part.

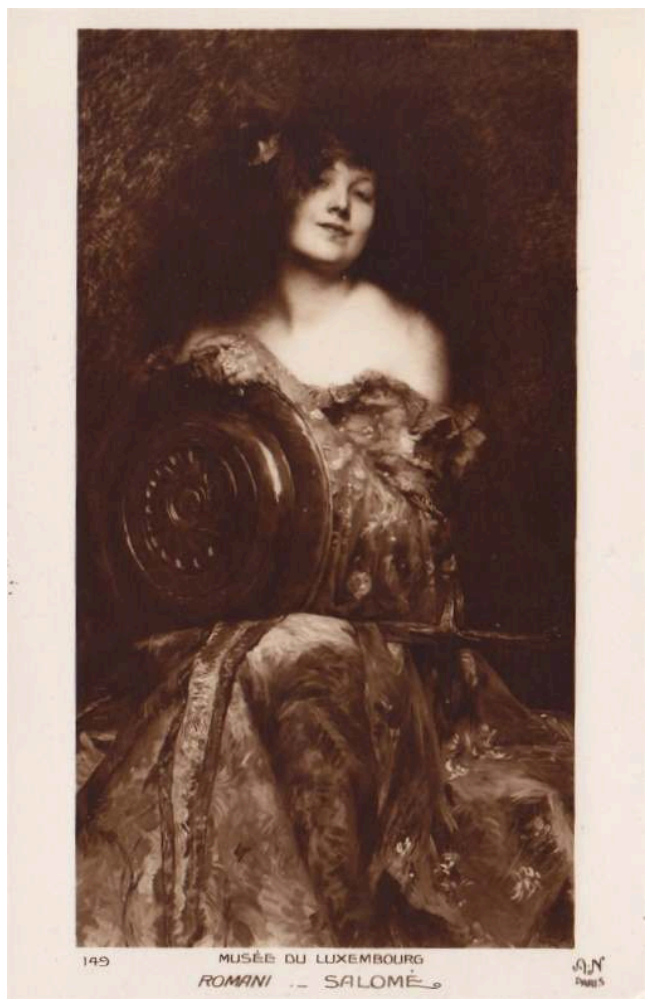

10 Les distinctions allaient généralement de pair puisque Boldini, décoré en 1889 d'une médaille d'or et élevé au rang de chevalier de la Légion d'honneur, était en 1900 récompensé du Grand prix. Grâce au succès commercial obtenu avec le marchandéditeur Adolphe Goupil ${ }^{5}$, Eduardo Tofano (1838-1920), habitué de la Société nationale des beaux-arts et du cercle de l'Union artistique, recevait, quant à lui, une médaille d'argent pour son portrait de Mlle Charcot. Une médaille d'or avait été enfin décernée pour le nocturne et introspectif En attendant la gloire (fig. 3) de Lionello Balestrieri (1872-1958) qui entamait une carrière parisienne et dont la notoriété allait être ramenée régulièrement à ce tableau. 
Fig. 3. - D'après Lionello Balestrieri, En attendant la gloire, 1897.

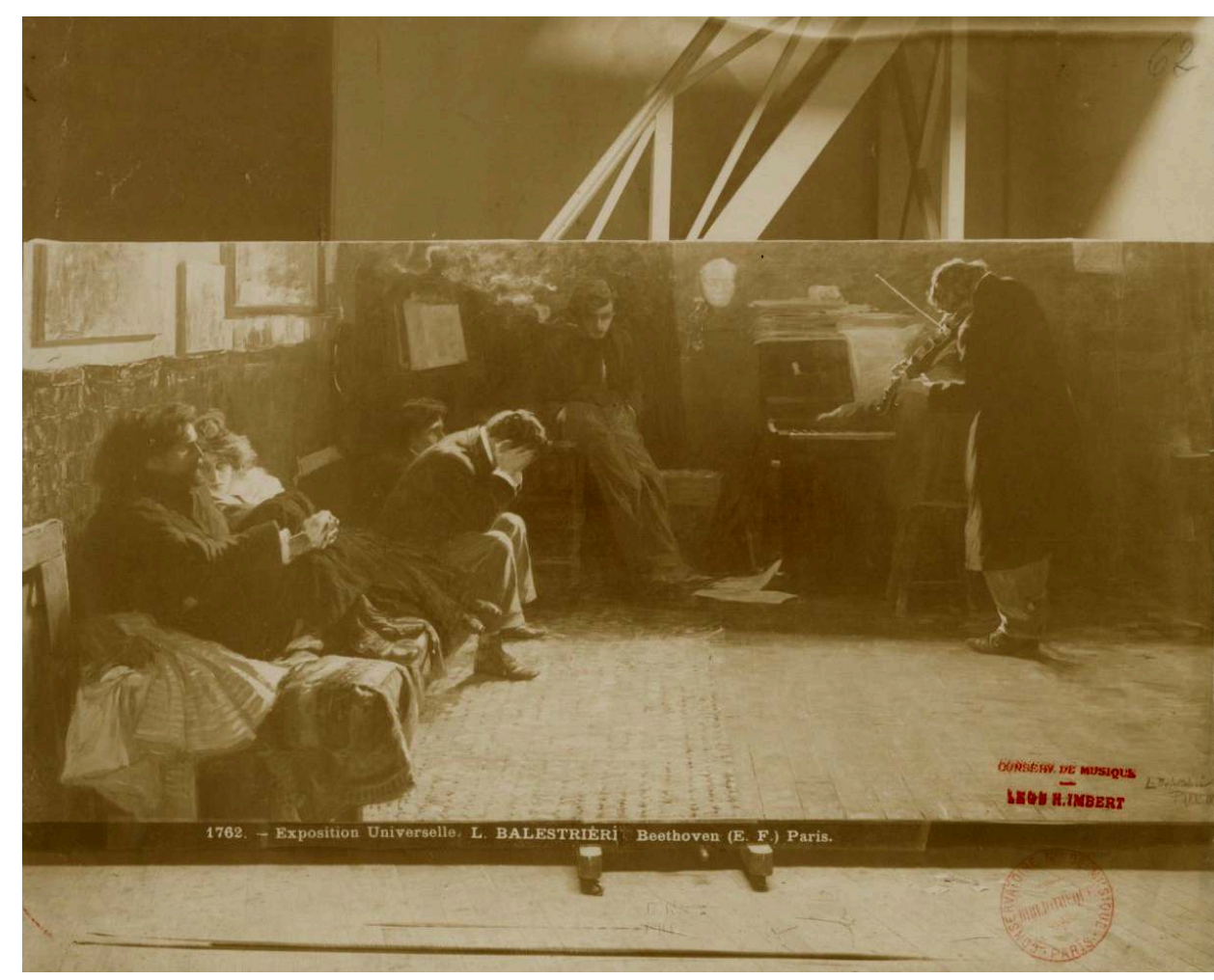

Tirage photographique présentant le tableau sur un chevalet mobile avant son accrochage à I'Exposition universelle [1900], 21,5x27 cm, Paris, Bibliothèque nationale de France.

(c) Source gallica.bnf.fr /BnF.

11 Malgré ce coup de projecteur sur les « archifrancisés » (Lafenestre, 1889 : 167), ceux-ci connaissaient une baisse drastique de leur participation. On en recensait uniquement huit lors de l'Exposition universelle de 1900 contre vingt lors de la précédente manifestation. En outre, l'accueil de la presse se révéla mitigé à leur égard. «Si M. Boldini et Mlle Juana Romani semblent avoir une esthétique commune, ils sont trop parisiens pour représenter des tendances générales » assenait-on (Quantin, 1900: 41). L'un des soutiens des artistes impressionnistes, Gustave Geffroy (1900), reprit le topos selon lequel l'artiste cosmopolite était étranger à tout caractère territorial spécifique. Boldini fut ainsi qualifié de "type d'artiste cosmopolite, adroit», et Geffroy de poursuivre: "nous voici plus loin que jamais de l'Italie ». L'avenir de l'art italien, tel que le concevait la critique française, n'était manifestement pas dans ces productions. Même Georges Lafenestre (1900), qui avait su apprécier en son temps le "Parisien » Giuseppe De Nittis (1846-1884), estima qu' «[...] on aura confiance dans l'évolution italienne vers un art plus indépendant, plus sain et plus fécond que celui des Italiens de Paris, de M. Boldini et Mme Juana Romani, dont la virtuosité, si brillante et habile qu'elle soit, même en produisant des chefs-d'œuvre [...] reste stérile pour leurs compatriotes. " Nous étions désormais loin des temps où le même critique (Lafenestre, 1889 : 140) célébrait cette influence française, cette supposée acculturation gage d'un progrès artistique :

Pour qui a pu voir l'exposition des artistes étrangers au palais Montaigne en 1855, pour qui se souvient de l'état d'abaissement dans lequel était alors tombé l'art de la peinture chez la plupart des peuples européens, même les plus glorieux par leur passé, tels que l'Italie, l'Espagne, la Hollande, et de l'état de barbarie dans lequel il 
se trouvait chez les peuples nouveaux, soit de l'Europe septentrionale, soit de l'Amérique, l'exposition actuelle, si incomplète qu'elle puisse être, montre de toutes parts, en l'espace de trente ans, une série d'étonnants progrès accomplis. [...] Sous

l'influence des exemples français, presque partout, les études techniques et historiques ont été renouvelées ou entreprises.

Le caractère apatride avait été même tenu en haute estime : «MM. De Nittis et Pasini sont eux aussi des nomades, italiens par le sang, mais français par le génie. À toutes ces seigneuries artistiques qui, la palette en main, ont fait irruption de ci de là dans notre monde, nous réservons des fauteuils sur une estrade cosmopolite [...]» (C. L., 1878). Charles Baudelaire (1868 : 212-213) n'avait-il pas parlé en 1855 de la "grâce divine du cosmopolitisme»?

La conception moderniste selon des canons "parisiens », pourtant encouragée par les Expositions universelles, ne correspondait manifestement plus aux attentes à un moment où les identités nationales se cristallisaient au sein du champ artistique. Même du point de vue de la critique parisienne, une "italianité", caractère artistique spécifique à un espace géographique, autrement dit une "nationalisation des styles " (Castelnuovo, 1987 : 995), devenait le gage de l'unité et de la cohésion de l'État-nation.

\section{Progrès artistique, unité et identité}

Ce changement de paradigme pour la scène artistique italienne s'opéra, aux yeux des observateurs parisiens, lors de l'Exposition universelle de 1900. Le déclin artistique, la " gloire passée » (Lafenestre, $1900: 292$ ), ne constituaient plus le poncif de la rhétorique critique. Au contraire, on considéra que l'Italie s'était mise « cette fois, résolument en marche " (Lafenestre, 1900 : 292). Cette appréciation inédite coïncidait tant avec le rayonnement récent des institutions et des manifestations italiennes, qu'avec le renouveau symbolique de Venise.

La mise en scène de la section d'art italien avait été pensée différemment que lors des éditions précédentes. Un chroniqueur parla d'une "une sorte d'anthologie fort intéressante de la peinture et de la sculpture italienne contemporaines» (L. E., 1900). On avait abandonné le modèle du Salon, où figuraient les seuls artistes contemporains, au profit de celui du musée (Ruedin, 2010: 83-86). La sélection des exposants de différentes générations, exception faite des «Italiens de Paris », se justifiait selon une double perspective :

Le commissariat général d'Italie vient de recevoir les œuvres choisies dans la Galerie nationale d'art moderne de Rome. Ce sont le Christ dans le désert, de Domenico Morelli; À l'étable, grande toile de Segantini; le Dyptique, de G.A. Sartorio ; la Gorgone et les héros et la Diane d'Éphèse, le grand succès de la dernière exposition internationale de Venise; le Viatique, de Morbelli, toile qui a donné tant de célébrité à l'artiste; Effet de lune de Mario de Maria, un des maitres de l'école vénitienne ; Coucher de soleil romain, de Carlandi ; Temps de pluie, de Henri Colemann; Dieu l'accompagne! de Faldi, de Florence ; de remarquables dessins du regretté Serra, chef de l'école bolognaise [...] (Anonyme, 1900b).

Les artistes désignés comme "chefs» de certaines écoles régionales historiques côtoyaient ceux reconnus par les récentes institutions artistiques italiennes et ayant, à ce titre, une "aura nationale». En acquérant des œuvres d'artistes exclusivement italiens, la Galleria Nazionale d'Arte Moderna, inaugurée en 1883 à l'occasion de l'Exposition internationale de Rome, constituait un modèle d'une structure artistique à 
l'échelle de la jeune nation. Cette unification du champ artistique s'était poursuivie par la fondation, en 1895, de l'Esposizione Internazionale d'Arte à Venise. Elle supplantait les expositions nationales des beaux-arts itinérantes dont la dernière se déroula, la même année, à Turin ${ }^{6}$. Débutée en 1897, la collection municipale d'art moderne de Venise, qui allait bientôt devenir la Galleria Internazionale d'Arte Moderna, complétait l'assise nationale et internationale de la scène artistique italienne. L'importance de la scène vénitienne dans ce panorama n'avait pas échappé au directeur du musée du Luxembourg, Bénédite, qui ne manqua pas de relever l'investissement soutenu (1904: 520-521) :

Venise est devenue aujourd'hui le centre le plus intense du mouvement artistique en Italie. Elle a ouvert une grande exposition internationale qui fonctionne tous les deux ans et qui en est à sa cinquième manifestation. Le chiffre des acquisitions qui ont été opérées en 1903 s'élève à près de quatre cent mille francs. C'est devenu le grand marché artistique de l'Italie. Un musée moderne a été créé par la municipalité et installé en 1900 au Palais Pesaro, légué par la duchesse BevilacquaLa Masa. Il comprenait avant les achats réalisés à l'exposition de 1903, près de 200 numéros.

17 Au-delà de cet aspect institutionnel, l'image de la cité lacustre se conjuguait alors avec une identité italienne en concordance avec le nouvel état-nation. L'« historique » école vénitienne incarnait par essence une histoire de l'art nationale susceptible de se rallier à une culture latine plus large (Jolivet, 2016). Or, le comité italien avait fait le choix d'un signal fort en faisant du pavillon (fig.4) un emblème de ce mythique imaginaire orientalisant de Venise, hybride du Palais des Doges et de la basilique Saint-Marc :

À l'entrée de la rue des Nations, sur le quai de la petite Venise parisienne qu'affleurent les gondoles un peu pesantes de la Compagnie des bateaux-mouches, se dresse le chef-d'œuvre du simili, le triomphe du triomphe du trompe-l'œil : le pavillon italien. Mosaïques, dorures, imitations de marbres polychromes, dentelures, balcons, clochetons; est-ce un palais, est-ce une église, est-ce un musée ? Chi lo sa? (Le Senne, $1900: 218$ ) 
Fig. 4. - Neurdein frères, Palais des nations, I'Italie, 1900.

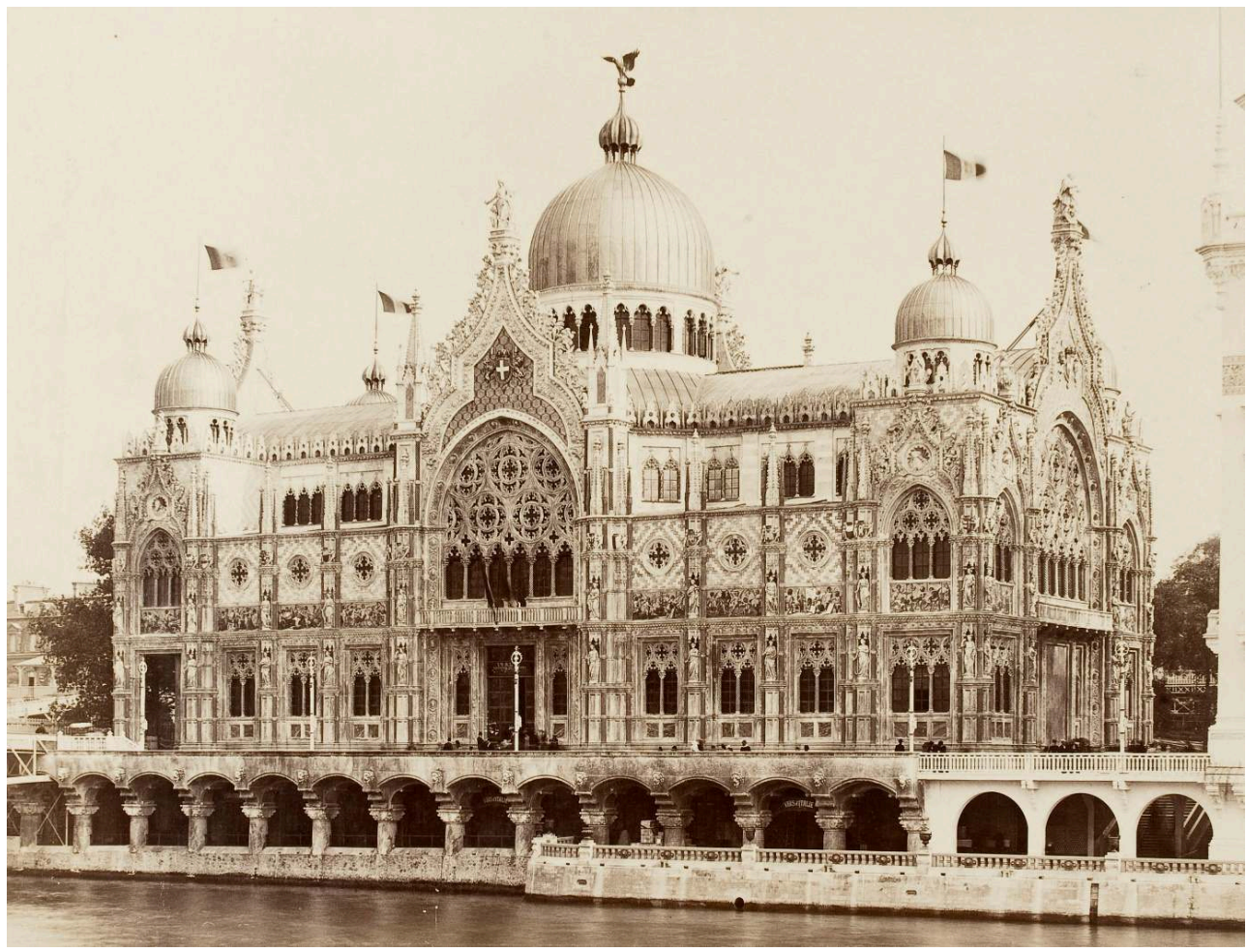

Tirage photographique contrecollé sur carton, 21,1×27,3 cm, Petit Palais, musée des Beaux-Arts de la Ville de Paris

ccø Paris Musées / Petit Palais, musée des Beaux-Arts de la Ville de Paris.

Ce « Palais des Allumettes », comme il avait été surnommé pour sa dimension factice, ne contenait pas pour autant la section des beaux-arts. L'attrait qu'il exerçait au prisme d'un imaginaire national faisait regretter aux contemporains cette privation d'une concordance entre l'objet et son écrin (Le Senne, 1900 : 218). La question d'un art unitaire était ainsi symboliquement ravivée. En effet, cette problématique avait animé les débats, depuis l'unification, tant chez les artistes que les critiques, lorsqu'ils s'étaient retrouvés en 1870 pour le premier congrès artistique organisé à Parme (Poppi, 1988). Une peinture moderniste, telle qu'elle se développait à Florence ou à Naples, avait constitué un temps une perspective intéressante de ralliement. Néanmoins, Camillo Boito (1873 : 418), critique d'art attitré de la Nuova Antologia, avait souligné l'intérêt de s'en remettre aux spécificités locales, sur l'exemple notamment des artistes vénitiens :

[C'est à] Venise, où les vieilles habitudes ont été finalement brisées, qui étaient plus tenaces que nulle part ailleurs en Italie, que sept ou huit jeunes gens ont amorcé l'étude ingénue, tenace, viscérale et individuelle de la nature, dans laquelle la chance veut qu'ils ne se ressemblent pas ${ }^{7}$.

Trente ans plus tard, Bénédite (1904:520) se rangeait derrière ces convictions, célébrant l'approche naturaliste du thème vénitien :

À Venise, tout un groupe d'observateurs compréhensifs, brillants et alertes, ou émus et recueillis, traduisent les spectacles variés à l'infini de cette ville de rêve où l'existence maritime du peuple offre à l'artiste toute son animation pittoresque et aussi toutes ses mélancolies. 
de l'Exposition universelle de 1900, Lafenestre, auteur d'un ouvrage d'histoire de l'art sur la peinture italienne et d'une monographie sur Titien (Lafenestre, 1855a et 1885b), reprit une idée similaire en l'adossant sur l'usage du terme d'école et sur la métaphore progressiste : « L'école vénitienne poursuit aussi, avec entrain, sa marche en avant [...]. C'est elle qui, jusqu'à présent, semble avoir le mieux profité des premiers exemples d'émancipation et de retour à la clarté et au charme pittoresque " (Lafenestre, 1900 : 293).

21 Toutefois, la reconnaissance de la peinture italienne dépassait le cadre de la Vénétie pour s'étendre à Milan et à Turin, deux autres pôles artistiques septentrionaux : « Si l'on juge par ce qui nous y est montré [au Grand Palais] de l'état présent de l'école [italienne], il semblerait que toute la vie et la vertu de l'art italien soient concentrées dans le nord de la péninsule. " (Michel, $1900: 2$ ) Ce «centre » incarnait l'« italianité » prisée des commentateurs à l'instar de Lafenestre $(1900: 294)$ à propos de peintres vénitien et milanais: "Les cinq tableaux de M. Ettore Tito [...], comme la Paix aux naufragés de M. [Leonardo] Bazzaro, sont des œuvres colorées, vivantes, expressives, vraiment italiennes ".

À l'inverse, les critiques, toujours prompts à vilipender les envois de petits formats jugés peu ambitieux, ne se satisfirent pas des compositions monumentales aux accents symbolistes de deux peintres «méridionaux ». L'« énigmatique et macabre " gorgone (Le Senne, 1900 : 219) de Giulio Aristide Sartorio (1860-1932) ou les « toiles démesurées insuffisamment remplies » (Geffroy, 1900) de Francesco Paolo Michetti (1851-1929) qui avait pourtant créé ces œuvres en vue de l'événement parisien, ne recueillirent pas l'assentiment de la presse. Lafenestre (1900 : 293) salua néanmoins chez le peintre des Abruzzes, autrefois familier du Salon, cette ambition à vouloir "prendre les allures d'un peintre épique et ethnique ». L'intérêt des recherches menées par Sartorio fut aussi discrètement relevé par Bénédite (1904: 524). Les acquisitions que ce dernier devait faire à l'Exposition universelle pour le musée du Luxembourg (Lagrange, 2021) en sa qualité de directeur, montrèrent qu'il privilégia des représentations évoquant un environnement septentrional, et ce dans un registre naturaliste. Furent ainsi achetés pour le compte de l'État, un paysage de plaine, non loin du lac majeur, du Milanais Filippo Carcano (1840-1914), une scène de rue (fig.5) du Vénitien Ettore Tito (1859-1941) et la toile plus singulière d'Angelo Morbelli (1853-1919), Jour de fête à l'Hospice Trivulzio, à Milan dont le réalisme était contrebalancé par l'irréalité de la lumière (Anonyme, 1901). 
Fig. 5. - D'après Ettore Tito, Chioggia, 1898, reproduit dans Revue de l'art ancien et moderne, $\mathrm{n}^{\circ} 40$, juillet, np.

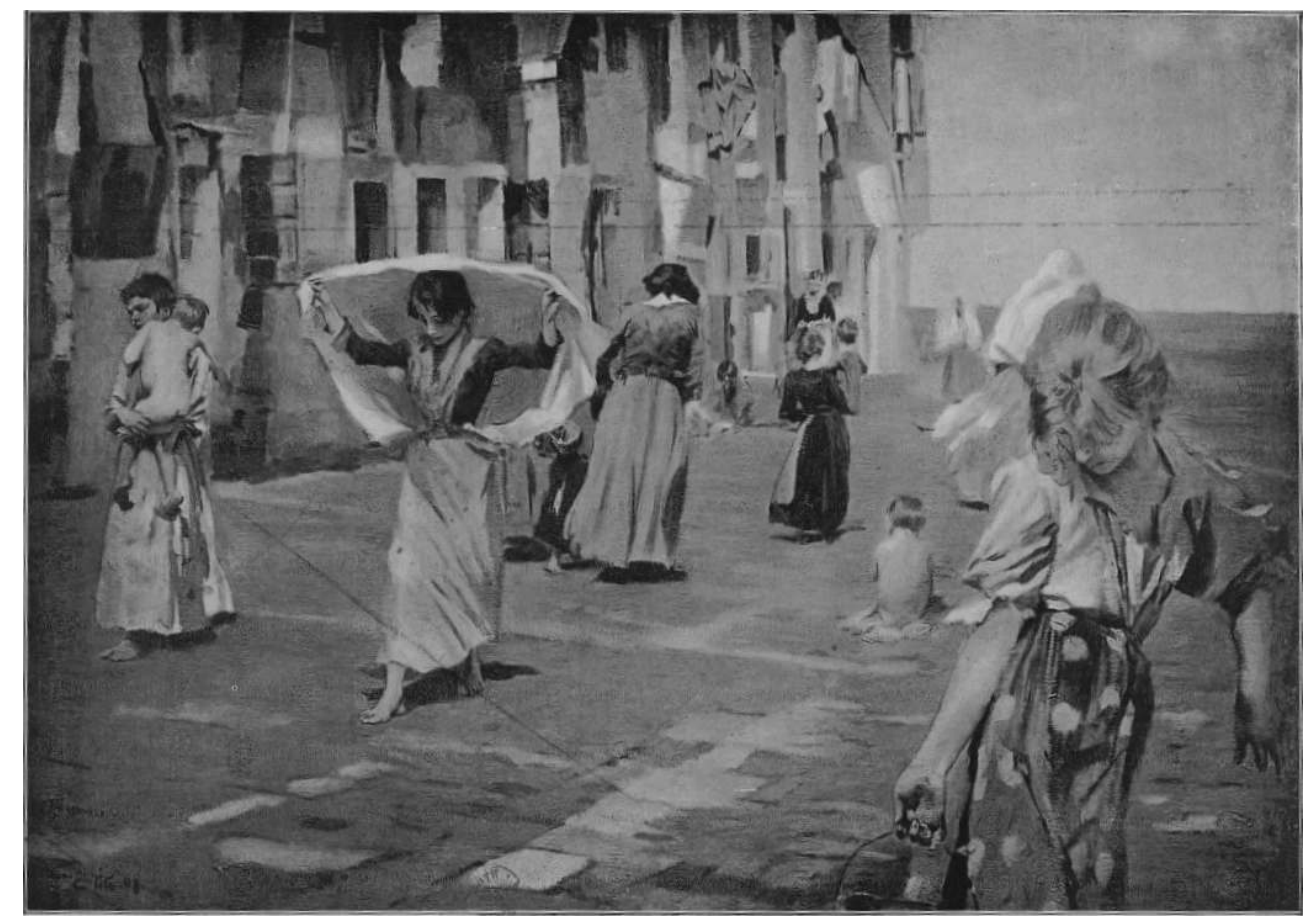

(C) Source gallica.bnf.fr /BnF.

Les œuvres de Michetti ou de Sartorio ne furent pas en revanche retenues alors que leurs auteurs avaient chacun bénéficié d'une salle particulière lors de la précédente Esposizione Internazionale d'Arte de Venise, signe de la reconnaissance qui leur était accordée en Italie.

L'image que se faisaient les critiques d'art français d'une peinture italienne reposait davantage sur l'idée d'écoles régionales porteuses de caractères supposés spécifiques à la jeune nation, tant dans leurs paysages que dans leurs «mœurs ». La valorisation du sujet, au détriment des aspects plus formels, conduisait inévitablement à conforter ces liens. Si l'école vénitienne devait sa popularité à ces aspects mais aussi à la renommée de ses manifestations artistiques et à l'édification du mythe italianisé de la Sérénissime, elle fut détrônée par la figure emblématique de feu Giovanni Segantini (1858-1899).

\section{Un apatride de l'art italien}

«Une grande victime de l'Art », ce fut ainsi que l'historien de l'art et critique Louis de Fourcaud (1900a), habituellement voué à l'art français, donna le ton de la réception de Segantini dans un article paru peu de temps après l'inauguration :

Au grand palais des Beaux-Arts de l'Exposition universelle, une salle entière de la section italienne est consacrée aux œuvres d'un peintre dont le nom, jusqu'ici, n'avait guère retenti en France. Il s'appelait Giovanni Segantini. Je dis qu'il s'appelait, car la mort l'a pris l'année dernière en pleine force de vie et de labeur, alors que commençait pour lui une renommée vraie. Ses compatriotes, fiers de son talent, ont voulu assurer à sa mémoire le rayonnement de l'hommage parisien. À nos yeux, par leurs soins, se sont groupés des tableaux alpestres où luisent, à la fleur des glaciers, les rayons bleus du matin, où flamboient parmi les crêtes les 
rayons ardents du midi qui pénètrent la terre verte des vallées, où resplendissent en éclats d'apothéoses les pourpres et les ors du soir.

D'une enfance solitaire et à bien des égards dramatique, Segantini était devenu un peintre en vue. Formé à l'Académie milanaise de Brera, il fut lié un temps avec le peintre et marchand Vittore Grubicy (1851-1920). Ce dernier l'avait notamment amené vers une esthétique divisionniste. La stricte approche naturaliste se trouvait réinterprétée au profit d'une perception panthéiste et symboliste des paysages, sans céder sur sa pratique du plein-air. Adepte des scènes rurales et des paysages de montagne, il s'était installé en Suisse en 1886, dans un village du nom de Savognin puis, à compter de 1894, à proximité des lacs d'Engadine. Il préparait « une de ses créations capitales » (Alexandre, 1900b : 5) pour l'Exposition universelle de 1900, La vie, la nature, la mort, lorsqu'il décéda brutalement, le 28 septembre 1899 . Ce n'est pas le moindre des paradoxes que de rappeler que le projet initial dans lequel le triptyque devait s'insérer - beaucoup plus ambitieux et financé sur fonds privé - était destiné au pavillon helvétique (Bücheler \& Lardelli, 1991). Bien qu'il pût se considérer comme Italien sans en avoir la nationalité (Quinsac, 2002: 259), son histoire personnelle expliquait vraisemblablement ses réticences à l'égard de l'instrumentalisation étatique de la culture (Ruedin, 2010 : 251).

Hors du Salon de 1884 et de l'Exposition universelle de 1889 où avaient figuré trois de ses tableaux, Chevaux, Vaches, Brebis, jugés alors anecdotiques, Segantini était peu connu en France, à l'exception «de quelques rares personnes»(Alexandre, 1900a: 3). Son travail suscitait en revanche un engouement dans les milieux sécessionnistes, à Berlin, Vienne et Munich. Ceux qui avaient porté un intérêt à son œuvre, Robert de la Sizeranne et William Ritter, figuraient parmi les auteurs français témoignant d'une sensibilité symboliste. La mort prématurée de l'artiste avait contribué à nourrir de nouveau le récit mythique de la vie du peintre des Alpes. En guise de nécrologie, une critique du journal féministe La Fronde (Péris, 1899) avait dressé un parallèle avec l'enfance du plus renommé des Primitifs italiens : "Ses débuts me rappellent ceux de Giotto : tous deux fils de pauvres campagnards, l'un commença sa carrière en gardant les moutons ; l'autre, pour gagner son pain, dut surveiller un troupeau de porcs. » L'héroïsation de Giotto avait été forgée par le récit de Giorgio Vasari (Kris \& Kurz, 2010 [1934] : 27-48) notamment au travers du caractère exceptionnel de son apprentissage. Une rhétorique similaire allait être en usage à propos de Segantini, contribuant à faire de lui, au-delà de tous les enjeux territoriaux, voire nationaux, un maître.

La section italienne accueillit en définitive une salle rétrospective composée du fameux triptyque inachevé (fig.6), de six autres toiles et de neuf dessins, la plupart préparatoires à ses plus fameuses compositions. 
Fig. 6. - Vue actuelle du triptyque La vie, La nature, La mort au Giovanni Segantini Museum, St. Moritz (Suisse).

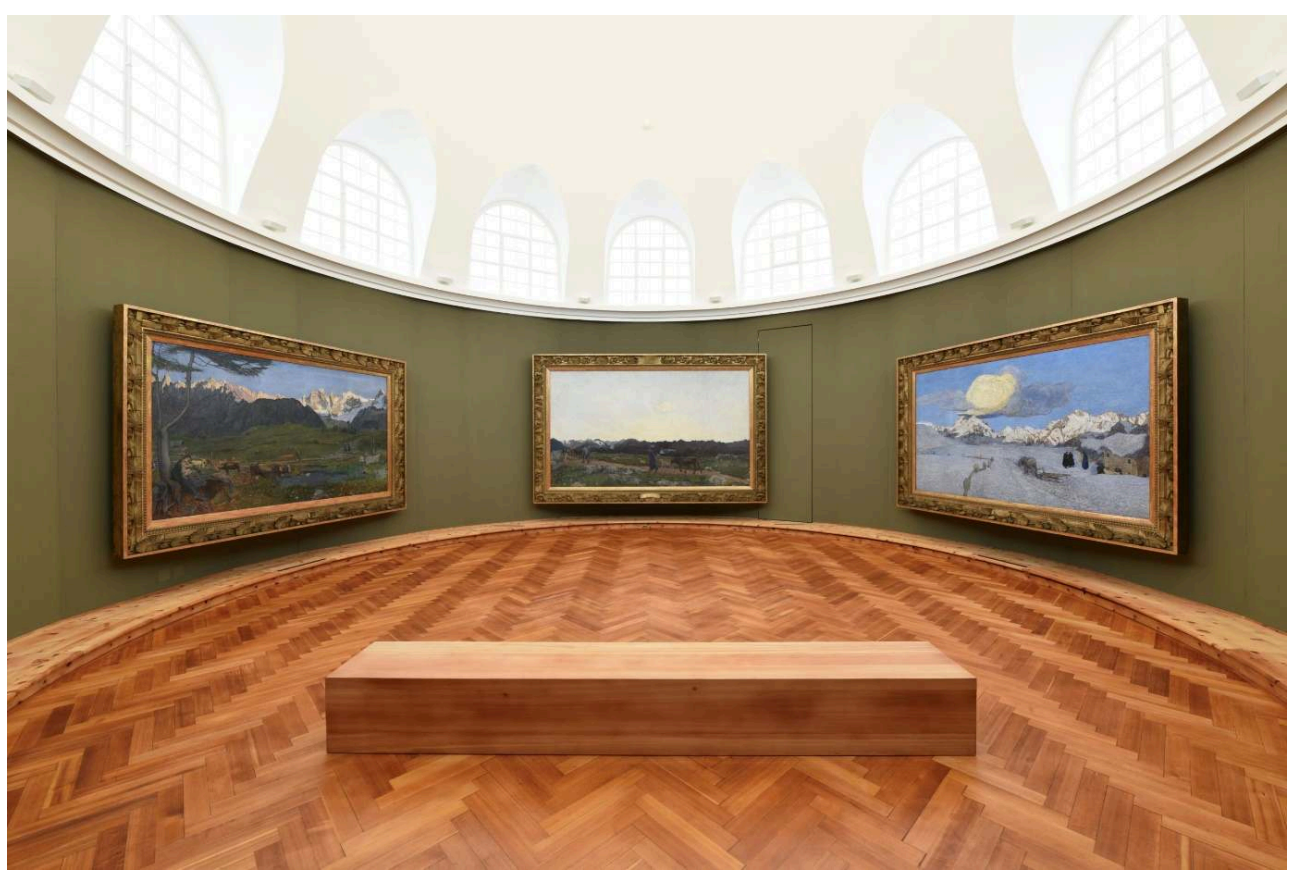

(c) Stephan Schenk, https://segantini-museum.ch/fotos-ausstellungsraum/.

29 L'accueil se révéla unanime. Tandis que le Figaro illustré faisait le choix de l'un de ses tableaux pour la couverture du numéro consacré à la section des beaux-arts (fig. 7), le critique Gustave Geffroy (1900) affirmait que «l'originalité de l'exposition italienne [était] chez Giovanni Segantini [...] ». 
Fig. 7. - Page de couverture de Figaro illustré, hors-série, novembre 1900, reproduisant II Frutto dell'amore [Fruit de l'amour] d'après Giovanni Segantini.

\section{FIGARO ILLUSTRE}

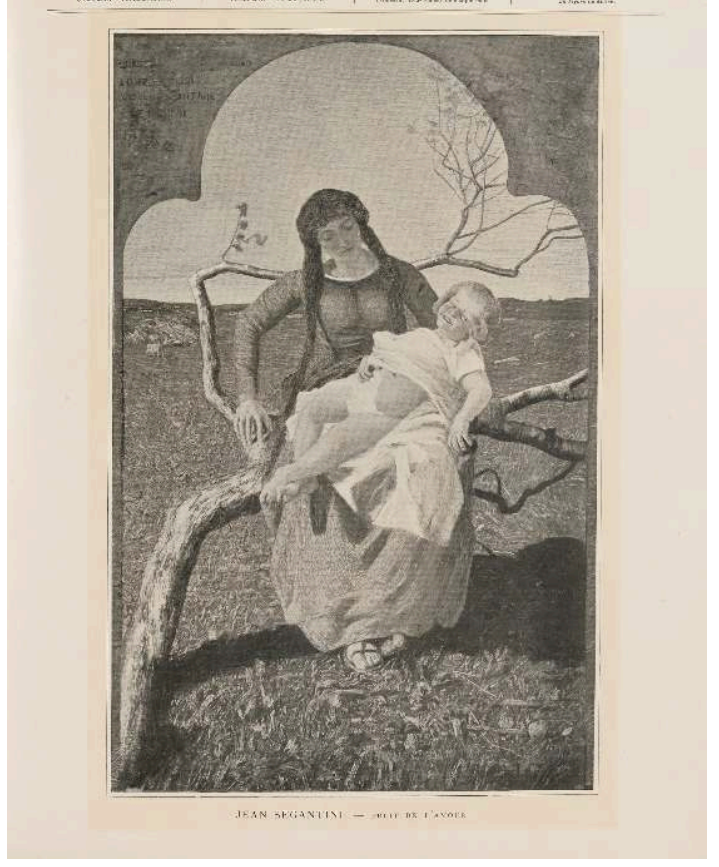

CCØ Paris Musées / Petit Palais, musée des Beaux-Arts de la Ville de Paris.

À son tour, André Michel (1900:2) assénait : «L'Italie a perdu en Segantini un maître volontaire, ardent, impérieux, une sorte de Millet montagnard plus sec et plus ligneux, qui se réfugia au cœur des Alpes neigeuses, loin des déclamations et des chansonnettes qui l'écœuraient. » Toutefois, dans les éloges prononcés, deux ambiguïtés pointaient. L'espace alpin, partagé par l'Italie, la Suisse, l'Autriche-Hongrie et la France, n'était guère marqué par une identité nationale spécifique. Loin d'être une rupture naturelle ou culturelle, les Alpes constituaient un territoire intermédiaire à la croisée de plusieurs cultures tout autant qu'un «espace d'unification» (Castelnuovo, 1987 : 999) générant sa propre identité transnationale. En outre, la critique parisienne tendait à faire de Segantini un "Millet italien» (Thiébault-Sisson, 1899) qui serait parti de « l'enseignement de l'école française » pour arriver à " une vision d'une rare puissance [...] où semblent se rencontrer les larges et robustes synthèses de Millet et, en même temps, l'acuité pénétrante des premiers Préraphaélites " (Bénédite, 1900: 586). Ce caractère singulier fit écrire à l'ardent défenseur de l'Art nouveau, François ThiébaultSisson (1899), quelques jours après la mort de l'artiste : «Et tout cela si peu italien, si septentrional, en somme, par le fond, d'un sentiment si contenu, d'une grâce si sévère et si forte ! C'est que l'artiste, à vrai dire, n'est italien que de nom. Il est né dans le Tyrol autrichien, à Arco, dans un pays de langue italienne, si l'on veut, mais d'une mentalité et d'une race où l'Italie n'y est pour rien. »

31 À l'unisson, Bénédite et Thiébault-Sisson paraissaient dénier tout prévalence de l'Italie sur l'art de Segantini. Comme si, mettre en exergue ces références françaises et anglaises, permettait de renouer avec l'idée d'une modernité à laquelle la peinture italienne ne pouvait pas avoir participé. 


\section{Conclusion}

L'ambivalence de la réception critique de la section italienne lors de l'Exposition universelle de 1900 constitua le creuset d'une rivalité entre une "italianité » et une modernité internationale (Metayer, 2012 : 201-226). Le retour sur le devant de la scène des écoles régionales - et plus particulièrement de la «nouvelle » école vénitienne aurait pu paraitre antinomique avec l'idée d'un art spécifiquement «italien». Néanmoins, c'est bien à partir de cette identité septentrionale que les critiques parisiens construisirent les principes d'une "italianité » à l'image du territoire alors que Venise concurrençait Rome comme "centre" artistique. Le raisonnement en "écoles" prévalait encore car il offrait la possibilité de définir, sur la base d'une supposée cohérence géographique, des ensembles de peintres, à défaut de pouvoir désigner un maitre spécifique à chaque "école ». L'engouement pour la peinture de Segantini s'inscrivit dans une dynamique inverse. Icône des transferts culturels européens malgré sa réclusion mythique dans les montagnes, il incarnait la figure du maitre, tout en demeurant une personnalité individualiste, allant à l'encontre de tout ce qui pourrait faire " école ». Les contradictions ne s'arrêtaient pas là. Sa peinture à l'image des "paysages artistiques" du territoire alpin n'en restait pas moins un paysage imaginaire, et surtout un paysage qui avait sa propre autonomie, paraissant échapper aux revendications identitaires nationales. Cet espace transfrontalier se trouvait ainsi défini comme un lieu de convergences des modernités européennes.

\section{BIBLIOGRAPHIE}

\section{Sources}

ALEXANDRE Arsène (1900a), « Les beaux-arts à l'Exposition. Espagne, Italie », Figaro, 16 juillet, 2-3.

ALEXANDRE Arsène (1900b), « Les beaux-arts à la Section Italienne du Grand Palais », Figaro illustré, hors-série, novembre 1900, 5-13.

ANONYME (1867), Liste du jury international, Paris : E. Dentu, 10.

ANONYME (1888), « L’Exposition de 1889 et le Monde artistique italien », L'Étendard (15), 21 mars, np.

ANONYME (1889), «Concours et expositions », Chronique des arts et de la curiosité, 16 mars, 82.

ANONYME (1900a), Journal officiel, 6 janvier, 69.

ANONYME (1900b), « Nouvelles », La Justice, 20 mars, 2.

ANONYME (1901), « Beaux-Arts », L'Intransigeant, 3 janvier, 3. 
BAUdelaire Charles (1868), « Exposition universelle. 1855. Beaux-Arts », Le Pays (mai-juin 1855) et Le Portefeuille (août 1855), repris dans Curiosités artistiques, Paris : Michel Lévy frères, 211-244.

BÉNÉDITE Léonce (1900), « Les Arts à l'Exposition universelle de 1900. L'Exposition décennales : la peinture étrangère ( $3^{\mathrm{e}}$ et dernier article), Gazette des beaux-arts, décembre, 577-592.

BÉNÉDITE Léonce (1904), « Italie », E. Picard (dir.), Exposition universelle internationale de 1900 à Paris (t. II), Paris : Lévy, 515-524.

BoIтo Camillo (1873), « Rassegna artistica », Nuova Antologia di science, lettere ed arti (vol. XXIV), Florence, 407-418.

BRÉMOND Jeanne (1900), « Les inaugurations d'hier. Le Palais de l'Italie », La Fronde, 3 mai, 1-2.

C. L. (1878), « Promenades à l'Exposition. II Les peintres italiens », Le Progrès de la Somme, 28 juillet, 1 .

FOURCAUd Émile de (1900), « Une grande victime de l'Art », Le Gaulois, 15 mai, 1.

GEFFROY Gustave (1900), «Les beaux-arts à l'Exposition. Les écoles étrangères de peinture », Le Journal, 28 mai, 3.

GuIDI Barbara [dir.] (2015), Boldini a Parigi. Ritratto di un pittore attraverso le lettere, Ferrare, Fondazione Ferrara Arte.

J. M. (1878), « Extérieur », Le Gaulois, 25 février, np.

LAFENESTRE Georges (1885a), La peinture italienne, t. 1 : Depuis les origines jusqu'à la fin du Xve siècle, Paris : A. Quantin.

LAFENESTRE Georges (1885b), La vie et l'œuvre de Titien, Paris : A. Quantin.

LAFENESTRE Georges (1889), « La Peinture étrangère à l'Exposition Universelle », Revue des Deux Mondes, $1^{\mathrm{er}}$ novembre, 138-172 (repris dans Artistes et Amateurs, Paris : Société d'édition artistique, s.d., 173-230).

LAFENESTRE Georges (1900), «L'Exposition universelle. La peinture. II. Les écoles étrangères », Revue de l'art ancien et moderne (40), juillet, 281-296.

L. E. (1900), « Notice concernant l'Italie à l'Exposition universelle de 1900 », Exposition internationale universelle de 1900. Volume annexe du Catalogue général officiel, Paris : Imprimeries Lemercier, Lille : L. Daniel, np.

LE SENNE Camille (1900), « Promenades esthétiques à l'Exposition universelle », Le Ménestrel, 15 juillet, 218-220.

Michel André (1900), « Causerie artistique. L'art à l'Exposition universelle », Journal des débats politiques et littéraires, 16 octobre, 1-2.

Milliot Lotty (1886), « L’État actuel de la peinture en Italie », La Nouvelle Revue (198), 349-364.

PÉRIS Louise (1899), « Un Deuil artistique en Italie », La Fronde, 5 octobre, 1.

QUANTIN Albert (1900), L'Exposition du Siècle, Paris, 41.

THIÉBAULT-ScISson François (1899), « Un Millet Italien. Giovanni Segantini », Le Temps, 6 septembre, 1. 


\section{Bibliographie}

BRUNO Silvia (2011), «Per essere io, volere o no, un antesignano » : la strategia culturale di Bartolomeo Bezzi tra Monaco e Venezia (thèse de doctorat en histoire de l'art), Università Ca'Foscari di Venezia. BÜCHELER Regula \& LARDELLI Dora [dir.] (1991), Giovanni Segantinis Panorama und andere Engadiner Panoramen, cat. exp. (St. Moritz, Segantini Museum, 17 juillet - 10 octobre 1991 ; Innsbruck, Landesmuseum Ferdinandeum, 17 octobre - $1^{\text {er }}$ décembre 1991), St. Moritz, Segantini Museum. CASTELnuovo Enrico \& GinzBuRg Carlo (1981 [1979]), « Domination symbolique et géographie artistique dans l'histoire de l'art italien ", Actes de la recherche en sciences sociales (40), novembre, 51-72 (traduction partielle de «Centro e periferia », G. Previtali [dir.], 1979, Storia dell'arte italiana, I. Questioni e metodi, Turin : Giulio Einaudi, 285-352).

CASTELnUovo Enrico (2014 [1987]), « La frontiera nella storia dell'arte », Il Capitale culturale. Studies on the Value of Cultural Heritage, numéro spécial Periferie. Dinamiche economiche territoriali e produzione artistica, 10, 985-1008, <http://riviste.unimc.it/index.php/cap-cult/article/view/938> ( ${ }^{\text {re }}$ éd. Ossola, Raffestin \& Ricciardi [dir.], 1987, « La Frontiera da Stato a nazione. Il caso Piemonte », Rome : Bulzoni, 234-261).

CESCHIN Daniele (2001), La « voce » di Venezia. Antonio Fradeletto e l'organizzazione della cultura tra Otto e Novecento, Padoue : Il Poligrafo.

DACOSTA KAUFMANN Thomas (2004), Toward a Geography of Art, Chicago et Londres : The University of Chicago Press.

Della ColetTA Cristina (2006), World's Fairs Italian Style. The Great Exhibitions in Turin and Their Narratives, 1860-1915, Toronto, Buffalo, Londres : University of Toronto Press.

FORSTER-HAHN Françoise (2003), « Ce que les Allemands ont présenté, ce que les Français ont vu. L'art allemand aux Expositions universelles de Paris de 1855 à $1900 »$ ", U. Fleckner Uwe \&

T. W. Gaehtgens (dir.), De Grünewald à Menzel. L'image de l'art allemand en France au XIXe siècle, 6, Paris : éditions de la Maison des sciences de l'homme.

GAMBONI Dario (1987), La géographie artistique (Ars Helvetica : arts et culture visuels en Suisse / Pro Helvetia), Disentis, Suisse : Ed. Desertina.

HARGRove June Ellen \& McWilliam Neil [dir.] (2005), Nationalism and French Visual Culture 1870-1914, Washington, National Gallery of Art ; New Haven, Londres : Yale University Press.

Holt GILMORE Elizabeth [dir.] (1982), The Art of All Nations : 1850-1873. The Emerging Role of Exhibitions and Critics, Princeton : Princeton University Press.

JoLIVET Anna (2016), L'Invention de l'école vénitienne en France au XIXe siècle, Rennes : Presses universitaires de Rennes.

KRIS Ernst \& KURZ Otto (2010), L'Image de l'artiste : légende, mythe et magie : un essai historique, Paris :

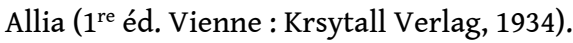

LAGRANGE Marion (2003), « Entre la maison Goupil et l'Italie, un axe commercial porteur d'une image identitaire », Histoire de l'art (52), juin, 121-134.

LAGRANGE Marion (2007), « Avant le Futurisme, les groupes artistiques italiens à Paris entre nécessités marchandes et sentiment national ", Recherches en histoire de l'art (6), 27-48. Lagrange Marion (2010), Les Peintres italiens en quête d'identité. Paris 1855-1909, Paris : INHA-CTHS. 
LAGRANGE Marion (2021, à paraître), « La salle italienne du musée du Luxembourg : histoire et limites d'une perspective nationale au début $\mathrm{du} \mathrm{xx}^{\mathrm{e}}$ siècle », A. Bonnet, P. chevalier \& F. Drugeon [dir.], Passages à Paris. Artistes étrangers à Paris de la fin du XIX siècle à nos jours, Paris : Mare\&Martin. LAMBERTI Maria Mimita (1982), « I mutamenti del mercato e le ricerche degli artisti », G. Bollati \& P. Fossati (dir.), Storia dell'arte italiana. Dal Medioevo al Novecento, 3, Il Novecento, Turin : G. Einaudi, $5-172$.

LAMBERTI Maria Mimita (2005), « Le mostre d'arte in Italia : gli studi recenti ed alcuni esempi », M. Hansmann \& M. Seidel (dir.), Pittura italiana nell'Ottocento, Venise : Marsilio, 179-198.

LAUGÉE Thierry \& RABILLER Carole [dir.] (2017), Critique d'art et nationalisme. Regards français sur l'art européen au XIX siècle, Bruxelles : P.I.E.-Peter Lang.

MAGGIO SERRA Rosanna (1991 [1990]), « I sistemi dell'arte nell'Ottocento », La Pittura in Italia. L'Ottocento, II, Milan : Electa (1 ${ }^{\text {re }}$ éd. 1990), 629-652.

METAYER Myriam (2012), Panoramas de l'art moderne. Manuels et synthèses en Italie et en France (1950-1970), Rennes : Presses universitaires de Rennes.

MILZA Pierre (1981), Français et Italiens à la fin du XIX siècle : aux origines du rapprochement francoitalien de 1900-1920, 2 vol., Rome : École française de Rome.

Peltre Christine \& LoRENTZ Philippe [dir.] (2007), La notion d'école, Strasbourg : Presses universitaires de Strasbourg.

Picone Petrusa Mariantonietta, Pessolano Maria Raffaela \& Bianco Assunta [dir.] (1988), Le grandi esposizioni in Italia 1861-1911: la competizione culturale con l'Europa e la ricerca dello stile nazionale, Naples : Liguori.

Picone PetRusA Mariantonietta (2015), « Le belle arti alle Grandi Esposizioni : uno sguardo alle presenze italiane (1851-1900) », B. costantino \& M. D’Agostino Manuela (dir.), Le Cattedrali dell'effimero. Viaggio bibliografico, iconografico e documentario attraverso le grandi esposizioni, cat. exp. (Naples, Accademia di Belle Arti, 29 octobre - 30 novembre 2015), Naples : Prismi, 43-62.

Pingeot Anne (2001), « 1880-1910, la sculpture italienne vue de Paris », G. Piantoni \& A. pingeot (dir.), Italies. 1880-1910 L'art italien à l'épreuve de la modernité, cat. exp. (Rome, Galleria Nazionale d'Arte Moderna, 22 décembre 2000 - 11 mars 2001, Paris, musée d'Orsay, 9 avril - 15 juillet 2001), Paris : Éditions de la Réunion des musées nationaux, 45-68.

Poppi Claudio (1988), « Il fantasma dell'arte unitaria : un viaggio attraverso le espozioni nazionali ", Il secondo '800 italiano. Le poetiche del vero, cat. exp. (Milan, Palazzo Reale, 26 mai - 11 septembre 1988), Milan : Gabriele Mazzotta, 58-73.

QUINSAC Annie-Paule (2002), « Trente-cinq ans d'études sur le divisionnisme italien : état de la question ", Perspectives (1), 242-272.

RoLAND Hubert \& SCHMITZ Sabine [dir.] (2004), Pour une iconographie des identités culturelles et nationales : la construction des images collectives à travers le texte et l'image. Ikonographie kultureller und nationaler Identität : zur Konstruktion kollektiver images in Text und Bild, Francfort-sur-le-Main : Peter Lang.

RUEDIN Pascal (2010), Beaux-Arts et représentation nationale : la participation des artistes suisses aux expositions universelles de Paris (1855-1900), 3, Bern : Peter Lang. 


\section{NOTES}

1. L'Histoire des peintres de toutes les écoles depuis la Renaissance jusqu'à nos jours de Charles Blanc a été édité sous forme de fascicules entre 1849 et 1876.

2. L'ouvrage issu de ma thèse de doctorat (Lagrange, 2010) n'effleurait, par rapport aux expositions universelles, que le sujet. En revanche, la problématique identitaire dans les expositions collectives italiennes à Paris a été approfondi (Lagrange, 2007).

3. La concordance avec l'anniversaire de la Révolution française avait conduit plusieurs monarchies à se dégager officiellement de cette manifestation pour laisser toute latitude à des comités indépendants. Les raisons étaient autres pour l'Italie. Si le rapprochement avec l'Empire allemand et l'Empire austro-hongrois officialisé depuis 1882, est souvent avancé, le motif principal résiderait dans l'échec de négociations commerciales ayant conduit à une "guerre douanière » (Milza, 1981).

4. Romani y exposait régulièrement depuis 1888, Boldini depuis 1877 et Eduardo Tofano depuis 1878.

5. Le tableau mentionné d'Eduardo Tofano fit l'objet, auprès de la maison Goupil, de dix modes de reproduction différents (Lagrange, 2003).

6. La chronologie avait été la suivante: 1861 (Florence), 1870 (Parme), 1872 (Milan), 1877 (Naples), 1880 (Turin), 1881 (Milan), 1883 (Rome), 1884 (Turin), 1887 (Venise), 1892 (Palerme) et 1895 (Turin).

7. [Traduction de l'auteur] «[È a] Venezia, dove, rotte finalmente le consuetudini vecchie dell'arte, ch'erano più tenaci in quella che non nelle altre provincie d'Italia, sette od otto giovani hanno iniziato lo studio ingenuo, tenace, viscerale e individuale della natura, nel quale la fortuna vuole che non si somiglino punto. »

\section{RÉSUMÉS}

Si les Expositions universelles de Paris avaient été durant la seconde moitié du XIX siècle, le théâtre de désapprobations à l'égard de la scène artistique italienne, les critiques lui reprochant sa décadence ou son cosmopolitisme dénaturé, l'Exposition universelle de 1900 amorce, par l'intermédiaire de son "palais vénitien", un renouveau qui prend son ancrage dans la territorialité des écoles italiennes. Malgré l'émergence d'un «État-nation » et une circulation accrue des artistes, le discours des critiques peine à se départir d'une historiographie de l'art italien, lui attribuant des caractères déterminés. Même les expositions internationales de Venise sont interprétées comme un signe de vitalité de l'« école vénitienne ». La notion d'école italienne se trouve alors moins investie que les écoles régionales qui paraissent maintenir des spécificités identitaires. Il n'est guère étonnant, dans ce contexte, qu'une figure telle que Giovanni Segantini, décédé l'année précédente, soit portée au faîte à l'encontre de personnalités italiennes installées à Paris, qui avaient été jusqu'alors les garantes d'une modernité.

If Paris World's Fairs had been the theatre of disapprovals towards the Italian artistic scene, critics blaming his decadence or his perverted cosmopolitism, 1900 World's Fair begin, with his "Italian Palace", a renewal taking his roots in Italian schools' territoriality. Despite the emergency of a "Nation State" and the increase of artists' circulation, critics had difficulties to give up the characteristics of an Italian art historiography. Even Venice's International 
Exhibitions are indirectly interpreted within the framework of the "Venitian School". The Italian school notion is in consequence less invested than regionals schools that seems maintain identitary speficities. So, in this context, it is not surprising that a figure like Giovanni Segantini, dead the last year, be carried up to the pinnacle, against Italian artists staying in Paris and guarantying a modernity.

Se le Esposizioni universali di Parigi erano state, nella seconda metà dell'Ottocento, il teatro delle disapprovazioni riguardo la scena artistica italiana di cui i critici ne biasimano la decadenza o il suo cosmopolitismo snaturato, l'Esposizione Universale del 1900 avvia, per mezzo del suo "palazzo veneziano», un rinnovamento che trova il suo ancoraggio nella territorialità delle scuole italiane. Nonostante l'emergere di uno «Stato-nazione» e una maggiore circolazione degli artisti, il discorso dei critici fatica a discostarsi da una storiografia dell'arte italiana, conferendole caratteristiche specifiche. Anche le esposizioni internazionali di Venezia vengono interpretate come un segno della vitalità della «scuola veneziana». La nozione di scuola italiana è quindi meno fortunata rispetto a quella delle scuole regionali che sembrano mantenere delle specificità identitarie. Non sorprende, in questo contesto, che un personaggio come Giovanni Segantini, scomparso l'anno precedente, venga acclamato come una celebrità, in opposizione alle personalità italiane residenti a Parigi che fino ad allora erano state garanti di una «modernità».

\section{INDEX}

Mots-clés : expositions universelles, histoires des expositions, école italienne, écoles régionales, géographie artistique

Parole chiave : Esposizioni universale, storia delle esposizioni, scuola italiana, scuole regionali, geografia del arte

Keywords : world's fairs, exhibitions history, italian school, regionals schools, geography of art

\section{AUTEUR}

\section{MARION LAGRANGE}

Maîtresse de conférences en histoire de l'art contemporain (Université Bordeaux Montaigne Centre François-Georges Pariset)

marion.lagrange@u-bordeaux-montaigne.fr 